\title{
Experimental Study on Inhibition Effects of the XAF1 Gene against Lung Cancer Cell Proliferation
}

\author{
Wen-Tao Yang ${ }^{1 \&}$, Dong-Lai Chen ${ }^{2 \&}$, Fu-Quan Zhang ${ }^{1 \&}$, Ying-Chen Xia ${ }^{1}$, Rong- \\ Ying Zhu ${ }^{1}$, Duan-Shan Zhou ${ }^{1}$, Yong-Bing Chen ${ }^{1 *}$
}

\begin{abstract}
Objective: To investigate the effect of high expression of XAF1 in vivo or in vitro on lung cancer cell growth and apoptosis. Methods: 1 . The A549 human lung cancer cell line was transfected with Ad5/F35 - XAF1, or Ad5/ F35 - Null at the same multiplicity of infection (MOI); (hereinafter referred to as transient transfected cell strain); XAF1 gene mRNA and protein expression was detected by reverse transcription polymerase chain reaction (RTPCR) and Western blotting respectively. 2. Methyl thiazolyl tetrazolium (MTT) and annexin V-FITC/PI double staining were used to detect cell proliferation and apoptosis before and after infection of Ad5/F35 - XAF1 with Western blotting for apoptosis related proteins, caspase 3, caspase - 8 and PARP. 3. After the XAF1 gene was transfected into lung cancer A549 cells by lentiviral vectors, and selected by screening with Blasticidin, reverse transcription polymerase chain reaction (RT-PCR) and Western blotting were applied to detect mRNA and protein expression, to establish a line with a stable high expression of XAF1 (hereinafter referred to as stable expression cell strain). Twenty nude mice were randomly divided into groups A and B, 10 in each group: A549/ XAF1 stable expression cell strain was subcutaneously injected in group A, and A549/Ctrl stable cell line stable expression cell strain in group B (control group), to observe transplanted tumor growth in nude mice. Results: The mRNA and protein expression of XAF1 in A549 cells transfected by Ad5/F35 - XAF1 was significantly higher than in the control group. XAF1 mediated by adenovirus vector demonstrated a dose dependent inhibition of lung cancer cell proliferation and induction of apoptosis. This was accompanied by cleavage of caspase $-\mathbf{- 3}, \mathbf{- 8 , - 9}$ and PARP, suggesting activation of intrinsic or extrinsic apoptotic pathways. A cell strain of lung cancer highly expressing XAF1 was established, and this demonstrated delayed tumor growth after transplantation in vivo. Conclusion: Adenovirus mediated XAF1 gene expression could inhibit proliferation and induce apoptosis in lung cancer cells in vitro; highly stable expression of XAF1 could also significantly inhibit the growth of transplanted tumors in nude mouse, with no obvious adverse reactions observed. Therefore, the XAF1 gene could become a new target for lung cancer treatment.
\end{abstract}

Keywords: XAF1 gene - A549 lung cancer cells - adenovirus - apoptosis - proliferation

Asian Pac J Cancer Prev, 15 (18), 7825-7829

\section{Introduction}

Lung cancer is a common malignant tumor in China, and NSCLC patients take up 80\% 85\% of total cases in lung cancer (Aydiner et al., 2013; Cai et al., 2013; Kaya et al., 2013; Mutlu et al., 2013; Natukula et al., 2013; Oven et al., 2013; Unal et al., 2013; Wang et al., 2013). How to effectively treat patients with lung cancer is a focus in clinical settings (Yan et al., 2009). Apoptosis plays an important role in the development of lung cancer (Yan et al., 2009). X-linked apoptosis inhibiting protein (XIAP) associated factor 1 (XAF1) is a new identified tumor suppressor genes, that is low expressed or even not expressed in many tumor tissue and tumor cell lines (Fong et al., 2000). This study investigated the effect of high expression of XAF1 with recombinant adenovirus as a carrier on inhibiting proliferation and inducing apoptosis in human lung cancer cell line A549 in vitro,, and used lentiviral vectors to build highly stable expression of XAF1 gene in lung cancer cell line, and injected lung cancer cell strains through the tail vein of nude mice to construct lung metastases model, finally provided experimental evidence for XAF1 gene therapy in vivo.

\section{Materials and Methods}

The XAF1 gene (Ad5/F35-XAF1) and controlled virus (Ad5/F35-Null) were provided by Beijing BenYuan Zhengang Company. lentiviral vectors carring XAF1 gene or null gene were bought from Shanghai Ruisai; 
Monoclonal thimble bought from Millipore Company; Reverse transcription kits bought from Promega Company; Blasticidin purchased from Sigma company. XAF1 antibodies bought from Abcam Company; PARP, Caspase 3 and Caspase - 8 antibodies bought from Cell Signaling Technology Company. Reference beta actin antibody was bought from Sigma Company.

\section{Experimental Animal}

Four-weeks old female BAI B/c nude mice were provided by Animal Experimental Centre of Shanghai Institutes for Sciences (Shanghai, China).

\section{Cell line}

Human lung adenocarcinoma cell line A549 (Ruijin Hospital affiliated to Shanghai Jiaotong University, Shanghai, China), was maintained in RPM1640 medium (Gibco, CA, USA) containing $10 \%$ fetal bovine serum (FBS). This cell line was maintained at $37^{\circ} \mathrm{C}$ and $5 \% \mathrm{CO}_{2}$.

\section{Construction of stable cell lines}

Lentiviral vectors carring XAF1 gene or null gene were transfected with lung adenocarcinoma A549 cells, screened with Blasticidin, constructed stable cell lines of A549/XAF1 and A549/Ctrl, and made A549/Ctrl as control.

\section{$R T-P C R$ (reverse transcription polymerase chain reaction)}

Total RNA was extracted from cells, total RNA was used for complementary DNA synthesis. Polymerase chain reaction was conducted. Product was recorded by gel imaging system and used for further analysis after electrophoresis.

\section{Western Blot analysis}

Total proteins were extracted, and protein samples were electrophoresed. The blots were incubated with specific primary antibodies, $4{ }^{\circ} \mathrm{C}$ for one night, and put with specific antibodies, kept $2 \mathrm{~h}$ at room temperature, Antigen-antibody complexes were visualized and imaged by

\section{MTT assay}

Cancer cells were seeded into 96-well plates at $1 \times 10^{4}$ cells/per well. After 24 hours, cells were infected with Ad5/F35-XAF1, Ad5/F35-Ctrl at different MOIs (10, 50 and 100), non-infected cells were the control group. After $48 \mathrm{~h}$ incubation, MTT was added to the cells and incubated for additional 4 hours. The absorbance (A value) was measured at the wavelength of 570 nanometers $(\mathrm{nm})$. Cell proliferation ratio $=($ experiment $\mathrm{A}$ value $-\operatorname{control} \mathrm{A}$ value)/control A value $\times 100 \%$. Repeated 3 times of each group experiment, and averaged.

\section{Flow cytometry}

Ad5/F35-NULL and Ad5/F35 - XAF1 were added to cell culture, according to the concentration of MOI 100 (method above), set up a control group (NOR). After $48 \mathrm{~h}$ incubation, a cell suspension was prepared. Add Annexin V-FITC and PI staining fluid, and delivered to flow cytometry to detect apoptosis rate. The experiments were repeated three times in each group, and achieved an average.

\section{Xenograft Mice Model}

Twenty four-weeks old female BALB/c nude mice were selected and were divided into two groups. Above mentioned two groups of stable cell line, with 1 x 106/0.1 $\mathrm{ml}$ phosphate buffer solution (PBS) was injected to right side back ribs of the nude mouse. Tumor size was measured using a caliper every five days. Animals were euthanized, and their tumors were weighed and harvested for histology analysis. Tumor volume (V) was calculated according to the following formula: $\mathrm{V}=\mathrm{V}=4 / 3 \pi \times \mathrm{L} / 2 \times$ $(\mathrm{w} / 2)^{2}$ (L: relatively shorter diameter, w: relatively longer diameter). Tumor specimens were taken photo, fixed by neutral formaldehyde, and paraffin embedded.

\section{Immunohistochemical analysis}

The tissue sections were deparaffinized, rehydrated and kept 30 minutes in $80{ }^{\circ} \mathrm{C}$ oven, incubated with anti- XAF1 (1:500), with peroxidase blockers overnight at $4{ }^{\circ} \mathrm{C}$ for antigen retrieval. Then, tumor sections were incubated with secondary antibodies. Then, add enough peroxidase substrate, use a microscope to control color, PBS to terminate reaction, stain nuclei with hematoxylin, and dehydration. Then to observe the color reaction of immunohistochemical slice (XAF1). Cells staining tan is positive.

\section{Statistics analysis}

SPSS 16.0 statistical software was used for analysis. analysis of variance (ANOVA) was used for comparison between univeriate factors, $p<0.05$ was regarded as statistically significant.

\section{Results}

XAF1 gene mRNA and protein expression in transient transfected lung cancer cell strain

According to PCR and Western blot analysis, after 48 h of Ad5/F35 - XAF1 transfection, XAF1 gene mRNA (Figure $1 \mathrm{~A}$ ) and protein expression in A549 cells (Figure 1b) increased significantly.

\section{XAF1 gene mRNA and protein expression in stable} expression cell strain

According to PCR and Western blot analysis, after Ad5/F35 - XAF1 transfection, XAF1 gene mRNA(Figure $2 \mathrm{a})$ and protein (Figure $2 \mathrm{~b}$ ) expression increased more

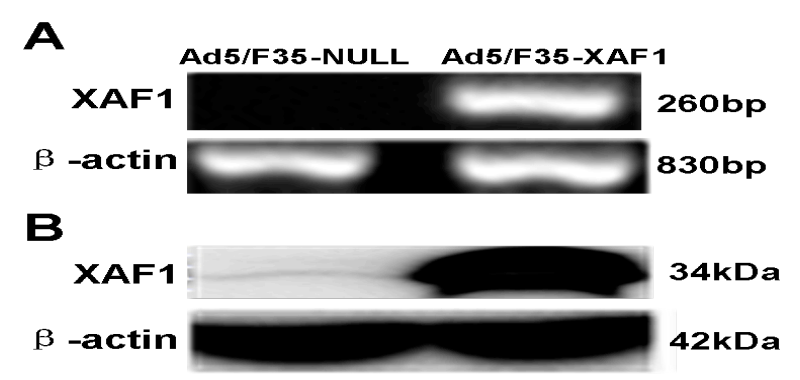

Figure 1. XAF1 Gene mRNA and Protein Expression in A549 Cell Increased Significantly after Adenovirus XAF1 Transfection 
XAF1

$\beta$-actin

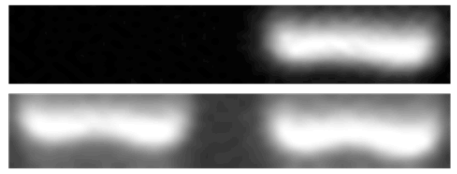

$260 \mathrm{bp}$

$830 \mathrm{bp}$

B

XAF1

A549/Ctrl

A549/XAF1

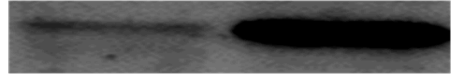

$\beta$-actin

$42 \mathrm{kDa}$

Figure 2. After Ad5/F35 - XAF1 Transfection, XAF1 Gene mRNA and Protein Expression Increased Significantly in A549/XAF1 Cell Lines

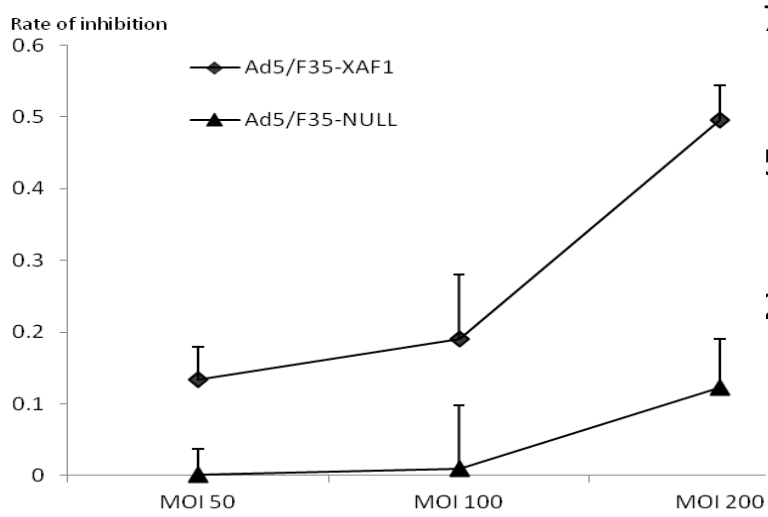

Figure 3. Transient High Expression of XAF1 Gene Significantly Inhibits the Proliferation of A549 Cells

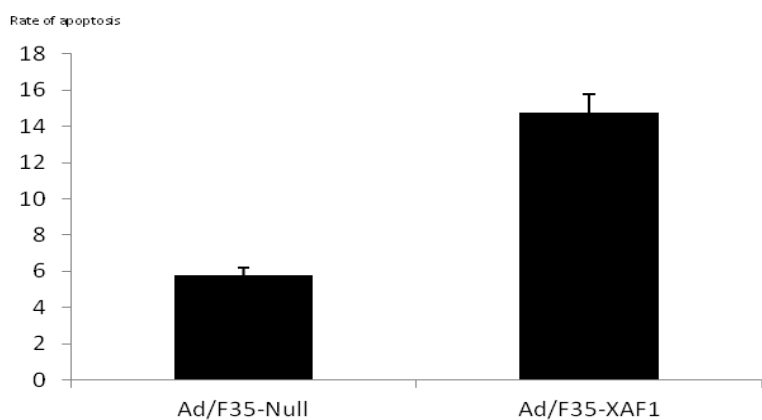

Figure 4. Transient High Expression of XAF1 Significantly Induced Apoptosis of A549 Cells

significantly in A549/XAF1 than in A549/Ctrl cell lines, suggesting that a cell line of A549 with a stable high expression of XAF1 gene is established.

\section{Cell proliferation}

By MTT method, it is suggested that with the increase of multiplicity of infection (MOI), cell proliferation rate of lung adenocarcinoma A549 gradually decreased. When the MOI reached 100 or 200, cell proliferation rate of Ad5/ F35 - XAF1 group was significantly lower than that of Ad5/F35 - Ctrl group ( $p<0.05)$ (Figure 3).

\section{Apoptosis}

Annexin V-FITC /PI double staining demonstrated that early apoptosis rate of A549 cells of Ad5/F35 - Ctrl transfection group was increased higher than that of Ad5/ F35 -XAFl group, and the difference was statistically significant $(p<0.05)$ (Figure 4$)$.

The expression of apoptosis related proteins

Compared with Ad5/F35 - Ctrl transfection group,
Ad5/F35-NULL Ad5/F35-XAF1

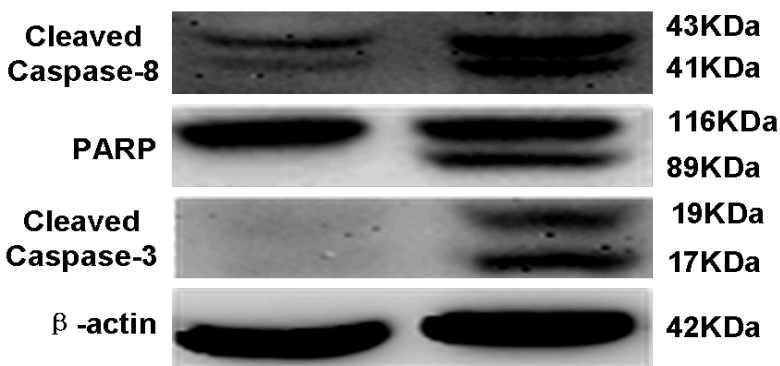

Figure 5. High XAF1 Gene Transient Expression Significantly Promoted the Cleavage of Apoptosis Related Caspases Protein

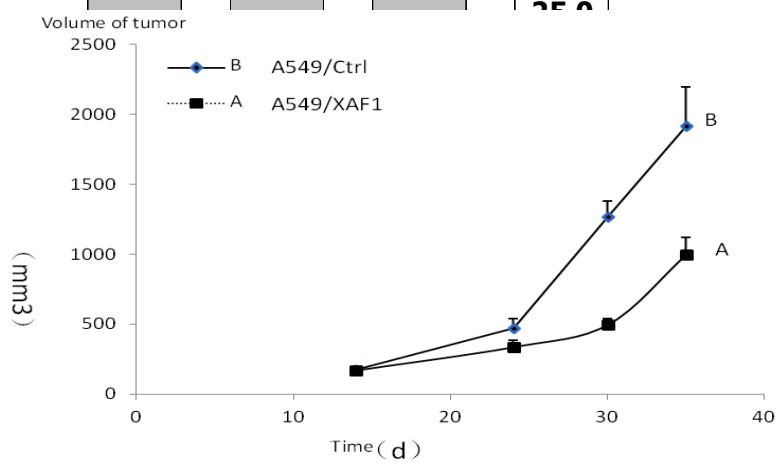

Figure 6. Consistently High Expression of XAF1 Gene Significantly Inhibit the Growth of Transplantation Tumor in Nude Mouse

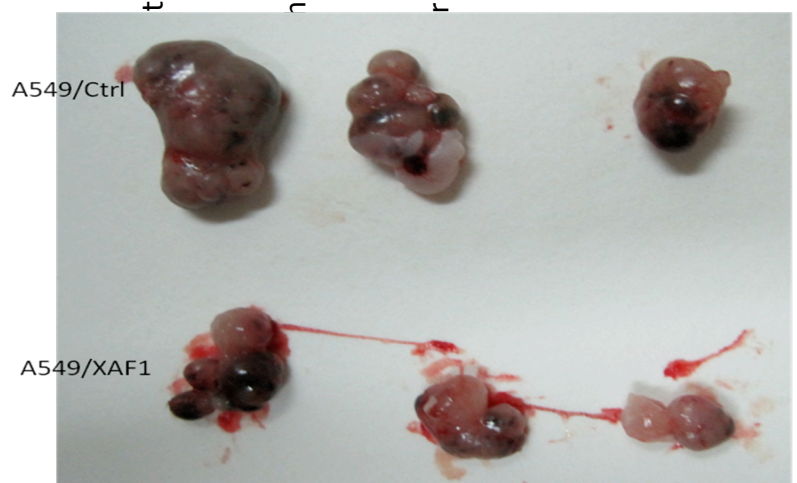

Figure 7. Tumor Sample of Subcutaneous Transplantation in Nude Mice

cleavage of PARP, Caspase 3 and Caspase- 8 protein was significantly demonstrated in Ad5/F35 - XAF1 transfection group of A549 cells, suggesting that caspase dependent apoptotic pathway is activated (Figure 5).

Transplanted tumor model of nude mice

Visible tumor tissue was formed 15 days after the injection to two groups of nude mice. Tumor tissue growth was significantly slower in A549/XAF1 group than that in A549/Ctrl group ( $p<0.05$, Figure 6 ) in a comparison when the diameter of subcutaneously transplanted tumor reached $1 \mathrm{~cm}$, and all mice were sacrificed (Figure 7).

\section{Expression of XAF1 protein on transplanted tumor tissue biopsies}

According to immunehistochemical analysis, XAF1 protein is expressed in cytoplasm and nucleus of lung metastatic tissue of A549/XAF1 nude mice (Figure 8). 


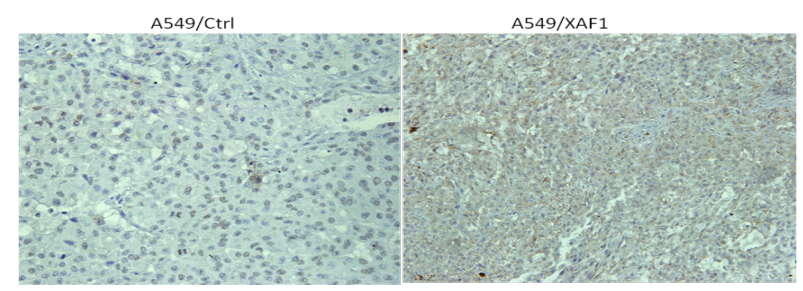

Figure 8. XAF1 Protein Expression was Significantly Increased in Lung Metastatic Tumor Tissue of Nude Mice That Were Injected with Cell Line A549/XAF1

\section{Discussion}

XAF1 is found by applying the method of yeast hybrid protein, a new type of XIAP antagonism, XAF1 can be directly combined with XIAP and have the effect of suppressing the antiapoptotic effects (Shibata et al., 2008; Kempken et al., 2009). Early experimental study shows that expression of XAF1 of lung squamous cancer tissues was significantly lower than that of normal lung tissue, and the expression of XAF1 in lung squamous carcinoma is associated with tumor stage, pathological grade, tumor invasion, lymph node metastasis, etc. of patients (Chen et al., 2011). But it is not clear whether high expression of XAF1 play a role in inhibiting lung cancer cell proliferation.

Viral vector is a gene carrier. It is the manipulating and transforming of the viral genome, and to make it to carry exogenous gene and the related components, and be packaged into virus particles. The virus vector that carrying exogenous gene is packaged into viral particles will constitute the gene delivery system. At present, Ad5 adenovirus type (type $\mathrm{C}$ virus) is commonly used as an adenovirus vector, with a wide range of host infection, could effectively proliferate in most human cells. Type B adenovirus (Ad35) microscopic protein generally exists in almost all cells (DC) on the surface of the membrane protein CD46 receptors, and it does not depend on the CAR and infect the target cells.

Rea et al reported that Ad35 fiber genes corresponding parts replaced by Ad5 vector all fiber genes or part of the fiber, it means that the fiber of virus Ad5 be converted into 35 adenovirus type fiber chimeric adenovirus vector, and established an Ad5/F35 adenovirus vector successfully (Rea et al., 2001). They changed the cell receptors from coxsackie adenovirus receptor (CAR) of Ad5 to CD46. Ad5/F35 adenovirus vector infected DC cells significantly and could be more effective than adenovirus vector Ad5 type, suggested that improving the ability of carrier infected cells in the treatment or prevention with carrier reduction is meaningful, is advantageous for the clinical application and reducing the toxicity. Therefore, Ad5/ F35 has good infectious in most of the cells of body, for a variety of hematopoietic cells (Yotnda et al., 2001; Itoh et al., 2003), stem cells, lymphocytes and tumor cells (Jung et al., 2005; Xiang et al., 2008). The infection efficiency is higher than ordinary adenovirus type 5, expression of exogenous gene with high quantity, without pathogenic ability to human body, and could gradually become one of the powerful tools for gene transduction.
In this study, we used recombinant adenovirus as a carrier, transient recover the expression of gene XAF1 in lung adenocarcinoma A549 cells in vitro, suggested that it was associated with inhibiting proliferation and inducing the apoptosis of tumor cells, mainly through apoptotic signaling pathways that depend on caspase. In this study, we use lentiviral vector as the carrier, to build a stable cell line of XAF1, and subcutaneous injected this tumor cell line in vivo to establish a nude mice model. Our results confirmed that the high stability of XAF1 expression could effectively inhibit the growth of lung cancer cells in nude mice transplantation tumor model.

In conclusion, restoring the expression of gene XAF1 in lung cancer A549 cells, could inhibit the proliferation of lung cancer cell, and significantly induce its apoptosis. The mechanism might be related to the signaling pathways activated by caspase apoptosis. XAF1 genes mediated by lentiviral, is associated with effectively expression, and could inhibit the growth of human lung cancer cells in nude mice tumor transplantation model. XAF1 gene is expected to become a new target for lung cancer treatment.

\section{Acknowledgements}

This study was supported by grants from Medical Research Foundation of Jiangsu Provincial Bureau of Health (NO. H201314), College Student Extracurricular Academic Foundation of Suzhou University (KY2014007Z), JunZheng Foundation of Soochow University (NO. 1230506139), Science and Technology Research foundation of Suzhou Municipality (NO. SYS201476).

\section{References}

Aydiner A, Ciftci R, Karabulut S, et al (2013). Does beta-blocker therapy improve the survival of patients with metastatic non-small cell lung cancer? Asian Pac J Cancer Prev, 14, 6109-14.

Chen YB, Shu J, Yang WT, et al (2011). XAF1 as a prognostic biomarker and therapeutic target in squamous cell lung cancer. Chin Med J, 124, 3238-43.

Fong W G, Liston P, Rajcan-Separovic E, et al (2000). Expression and genetic analysis of XIAP-associated factor 1 (XAF1) in cancer cell lines. Genomics, 70, 113-22.

Itoh A, Okada T, Mizuguchi HA, et al (2003). Soluble CARSCF fusion protein improves adenoviral vector-mediated gene transfer to c-Kit-positive hematopoietic cells. J Gene Med, 5, 929-40

Jung D, Néron S, Drouin M, et al (2005). Efficient gene transfer into normal human B lymphocytes with the chimeric adenoviral vector Ad5/F35. J Immunol Methods, 304, 78-7

Kempken Steffen C, Fritzsche FR, Johannsen M, et al (2009). Down-regulation of the pro-apoptotic XIAP associated factor-1 (XAF1) during progression of clear-cell renal cancer. BMC Cancer, 9, 276.

Oven Ustaalioglu BB, Unal OU, Turan N, et al (2013). Prognostic factors for lymph node negative stage I and IIA non-small cell lung cancer: multicenter experiences. Asian Pac J Cancer Prev, 14, 6287-92.

Rea D, Havenga MJ, van Den Assem M, et al (2001). Highly efficient transduction of human monocyte-derived dendritic cells with subgroup B fiber-modified adeno-virus vectors enhances transgene-encoded antigen presentation to 
cytotoxic T cells. J Immunol, 166, 5236-44

Schroers R, Hildebrandt Y, Hasenkamp J, et al (2004). Gene transfer into human $\mathrm{T}$ lymphocytes and natural killer cells by Ad5/F35 chimeric adenoviral vectors. Exp Hematol, 32, 536-46

Shibata T, Noguchi T, Takeno S, et al (2008). Disturbed XIAP and XAF1 expression balance is an independent prognostic factor in gastric adenocarcinomas. Ann Surg Oncol, 15, 3579-87.

Xiang DB, Chen ZT, Wang D, et al (2008). Chimeric adenoviral vector Ad5/F35- mediated APE1 siRNA enhances sensitivity of human colorectal cancer cells to radiotherapy in vitro and in vivo. Cancer Gene Ther, 15, 625-35.

Yan F, Gou L, Yang J, et al (2009). A novel pro-apoptosis gene PNAS4 that induces apoptosis in A549 human lung adenocarcinoma cells and inhibits tumor growth in mice. Biochimie, 91, 502-7.

Yotnda P, Onishi H, Heslop HE, et al (2001). Efficient infection of primitive hemato- hematopoietic stem cells by modified adenovirus. Gene Ther, 8, 930-37 